\title{
APPLICATION OF NOISE RESISTANCE IN CARBON STEEL CORROSION
}

Y.F. Cheng ${ }^{\mathrm{a}, *}$, M. Wilmott ${ }^{\mathrm{b}}$ and J.L. Luo ${ }^{\mathrm{c}}$

a NOVA Research and Technology Center, 2928- 16 Street N.E., Calgary, Alberta, T2E 7K7, Canada $b$ Bredero Shaw, Imperial Dock, Leith, Edinburgh, Scotland EH6 7DT, UK

c Dept. of Chemical and Materials Engineering, University of Alberta, Edmonton, Alberta, T6G 2G6, Canada

Abstract

Electrochemical noise measurements and impedance analyses were performed on A516-70 carbon steel in chromate/chloride solutions. The noise data were used to calculate the noise resistance in the time domain, and the spectral noise resistance and spectral noise impedance in the frequency domain. It is shown that the spectral noise impedance is equivalent to the modules of electrode impedance over the experimental frequency range. This relationship is independent of the corrosion types (passivity, general corrosion and metastable pitting). The noise resistance coincides with the charge-transfer resistance and the spectral noise resistance only under certain conditions, such as passive state or general corrosion, where the noise resistance provides an indication of the corrosion resistance of the material under study. During pitting corrosion, the noise resistance is much lower than the charge-transfer resistance and only qualitatively, rather than quantitatively, tracks the change of corrosion resistance. The spectral noise resistance is consistent with the charge-transfer resistance and can be used to indicate the corrosivity of carbon steel under studied conditions.

Keywords: electrochemical noise, noise resistance, spectral noise resistance, spectral noise impedance, electrode impedance, charge-transfer resistance, carbon steel

Introduction

The measurement and analysis of electrochemical noise (EN) have become widespread for obtaining corrosion information [1-7]. Of the various methods for analysis of EN data, the application of noise resistance $\left(R_{n}\right)$ has recently seen a growing interest [8-15]

$\mathrm{EN}$ is generally measured as potential and current fluctuations in a time series. The current is measured

between two nominally identical working electrodes (WE) connected through a zero resistance ammeter 
(ZRA). The potential measurement is made between the ZRA coupled electrodes and a third reference electrode, which is either a conventional reference electrode or an electrode of the same material as the WEs. The noise resistance is calculated as the ratio of the standard deviation of potential to the standarc deviation of current over a fixed period of time $[10,14]$. Theoretical analysis has shown that the potential and current noise are related by the impedance of the metal-solution interface [16]. It is proposed that noise resistance can be interpreted in essentially the same way as conventional chargetransfer resistance $[16,17]$. Obviously, noise resistance is a function of the electrochemical behavior of the material under study.

The noise data can also be transformed from the time domain into the frequency domain to obtain power spectral density (PSD) using either fast Fourier transform (FFT) [18] or the maximum entropy method (MEM) [19]. The transformed potential and current data at each frequency can be used to calculate the spectral noise impedance $\left(\mathrm{R}_{\mathrm{sn}}(\mathrm{f})\right)$, the square root of the ratio of potential PSD to current PSD, at each frequency. Similar to the relationship between noise resistance and charge-transfer resistance, the spectral noise impedance should have some analogy with the modulus of the electrochemical impedance $(|z|)$.

Noise resistance has been used for many aspects in corrosion. For example, it has been used to study coating performance and degradation, to determine corrosion rate and to study the onset of localized corrosion. Many researchers have shown $[8-10,20]$ that noise resistance can be used to qualitatively monitor the changes in the corrosion resistance. However, there are still arguments on relationship between $R_{n}$ and charge-transfer resistance $\left(R_{p}\right)$. For example, Dawson et al. [21] found that $R_{n}$ correlated well with both the charge-transfer resistance and weight loss of carbon steel in acid, while the analysis results for 310 stainless steel in chloride solutions presented by Kelly et al. [20] indicated that $R_{n}$ generally overestimated the charge-transfer resistance, leading to an underestimate of the corrosion rate. Although some attempts to justify the relationship between $R_{n}$ and charge-transfer resistance on the theoretical basis have been published [22], their validity has been questioned [23,24]. Chen and Bogaert [25] proposed a mathematical derivation based on the kinetics of the interfacial processes, however, its applicability was restricted because the mathematical treatment required appropriate assumptions such as activation-controlled anodic and cathodic reactions. Bertocci and Huet [3] described a model linking $R_{n}$ and the impedance modulus of the electrode, $|z|$, by computer simulation. The model still needs further experimental verifications.
Recently, Bertocci et al. [14] presented a model of the current and potential fluctuations simultaneously occurring in an electrochemical cell based on either current or potential noise source internal to the electrodes. This model allows the evaluation of $R_{n}$ and $R_{s n}(f)$, and the development of the theoretical basis for the relationship between $R_{n}$ and the electrode impedance, considering the various measurement schemes and affecting parameters such as cell geometry, solution resistance and electrodes with different kinetics. It was showed that, over a quite large range of conditions, $R_{\mathrm{sn}}(f)$ coincides with the impedance modulus $|Z|$ of the electrode. The $R_{n}$ is equal to the charge-transfer resistance $R_{p}$ if the electrode impedance is equal to $R_{p}$ in the frequency bandwidth investigated. The further experimental results [15] showed that the above derivation does not depend on the corrosion kinetics and can be valid for localized corrosion as well as general corrosion.

In the present work, the measurements of EN and electrochemical impedance were performed for A516-70 carbon steel in different corrosive environments designed to set up either passivity, general corrosion or pitting. Because the charge-transfer resistance, obtained by impedance measurements, is valid to reflect the corrosion resistance for both general corrosion and localized corrosion systems, the noise resistance, spectral noise impedance and spectral noise resistance were calculated and compared with the charge-transfer resistance and electrode impedance. The theoretical basis of the application of noise resistance for carbon steel corrosion in various solutions is discussed.

\section{Experimental}

\section{Electrodes and solutions}

Coupon specimens of $15 \mathrm{~mm} \times 15 \mathrm{~mm} \times 5 \mathrm{~mm}$ were cut from a sheet of A516-70 carbon steel with chemical composition (\%, wt): C 0.31, Mn 1.0, P 0.035, S 0.04 and Si 0.25 . The surfaces of specimens were ground with emery paper to a 600 grit finish. In order to prevent crevice corrosion the samples were coated with a layer of masking paint, and then embedded in an epoxy resin leaving an area of 0.4 $\mathrm{cm}^{2}$ exposed to solution. The working surface was polished with emery paper to a 1,000 grit finish, cleaned in distilled water and degreased in methanol.

When the carbon steel specimens were immersed in $0.05 \mathrm{M} \mathrm{Na}_{2} \mathrm{CrO}_{4}$ and $0.05 \mathrm{M} \mathrm{NaCl}$ solutions, stable passivity and general corrosion conditions, respectively, were reached after a certain time. In order to investigate the pitting behavior of carbon steel, chloride ions were added to the $0.05 \mathrm{M} \mathrm{Na}_{2} \mathrm{CrO}$ 
solution to produce $0.1 \mathrm{M} \mathrm{Cl}$. All the solutions were made from analytical grade reagents and distilled water. The tests were carried out at ambient temperature.

\section{Electrochemical noise measurements}

During the measurements of current noise, a pair of nominally identical A516-70 carbon steel specimens, used as the WEs, were immersed in the testing solutions. $\mathrm{A} \mathrm{Ag} / \mathrm{AgCl}$ electrode was employed as the reference electrode. The current noise data were recorded using an ACM Auto ZRA measurement system with a sampling rate of 1 point per second.

\section{Electrochemical noise analysis}

The noise data collected in the time domain were transformed into the frequency domain to obtain PSD by FFT [18]. Trend removal was performed to remove the direct current shift contained in the current noise when calculating the PSD. The PSD frequency range was determined by the sampling interval, $\Delta t$, and the number of points, $\mathrm{N}$, in each calculating cycle. In this work the sampling rate was $1 \mathrm{~Hz}$ and there were 1,024 points in each calculating cycle. Therefore, the experimental frequency range of PSD was:

$$
\begin{aligned}
& f_{\text {min }}=\frac{1}{N \Delta t}=\frac{1}{1024 \times 1} \approx 10^{-3} \mathrm{~Hz} \\
& f_{\text {max }}=\frac{1}{2 \Delta t}=\frac{1}{2 \times 1}=0.5 \mathrm{~Hz}
\end{aligned}
$$

The noise resistance, $R_{n}$, and spectral noise impedance, $R_{\mathrm{sn}}(\mathrm{f})$, were statistically calculated in the time and frequency domains, respectively, according to the following equations [8-10,13]

$$
\begin{gathered}
R n=\frac{\sigma E(t)}{\sigma I(t)} \\
R_{S n}(f)=\left|\frac{E_{P S D}(f)}{I_{P S D}(f)}\right|^{\frac{1}{2}}
\end{gathered}
$$

where $\sigma E(t)$ and $\sigma I(t)$ are the standard deviations of potential and current, respectively. $E_{P S D}(f)$ and $I_{\text {PSD }}(f)$ are the PSD data of potential and current fluctuations, respectively. The spectral noise resistance $\mathrm{R}_{\mathrm{sn}}{ }^{0}$ is defined as the zero frequency limit of the spectral noise impedance [8,9]

$$
R_{\mathrm{sn}}{ }^{0}=\lim _{f \rightarrow 0} R_{\mathrm{sn}}(f)
$$

Electrochemical impedance measurements

Electrochemical impedance spectroscopy (EIS) measurements were performed for the specimens immediately after the noise monitoring using a Gamry CMS 300 corrosion measurement system. The A516-70 carbon steel was employed as the $\mathrm{WE}, \mathrm{a} \mathrm{Ag} / \mathrm{AgCl}$ electrode as the reference electrode, and a coiled platinum wire as the counter electrode. The test frequencies ranged from $5,000 \mathrm{~Hz}$ to $0.005 \mathrm{~Hz}$. The values of charge-transfer resistance $R_{t}$ were determined by impedance measurements.

Results

\section{Carbon steel in chromate solution}

Polarization curves for A516-70 carbon steel exposed to each of the test solutions are shown in Fig. 1. It is obvious that the stable passivity of $\mathrm{A} 516-70$ carbon steel is established in $0.05 \mathrm{M} \mathrm{CrO}_{4}{ }^{2-}$ solution The passive potential ranged from $-400 \mathrm{mV}$ to $950 \mathrm{mV}$. Chromate is an oxidizing inhibitor for steels. A sufficiently high concentrations, chromate contributes to the formation of a uniform and protective surface oxide film $[26,27]$. When chromate adsorption predominates on the surface of a specimen passive films of $\mathrm{Cr}_{2} \mathrm{O}_{3}$ or $\mathrm{Cr}(\mathrm{OH})_{3}$ can be formed according to the competitive adsorption model proposed by McCafferty [26]. Therefore, A516-70 carbon steel in sodium chromate solution is considered a passive system.
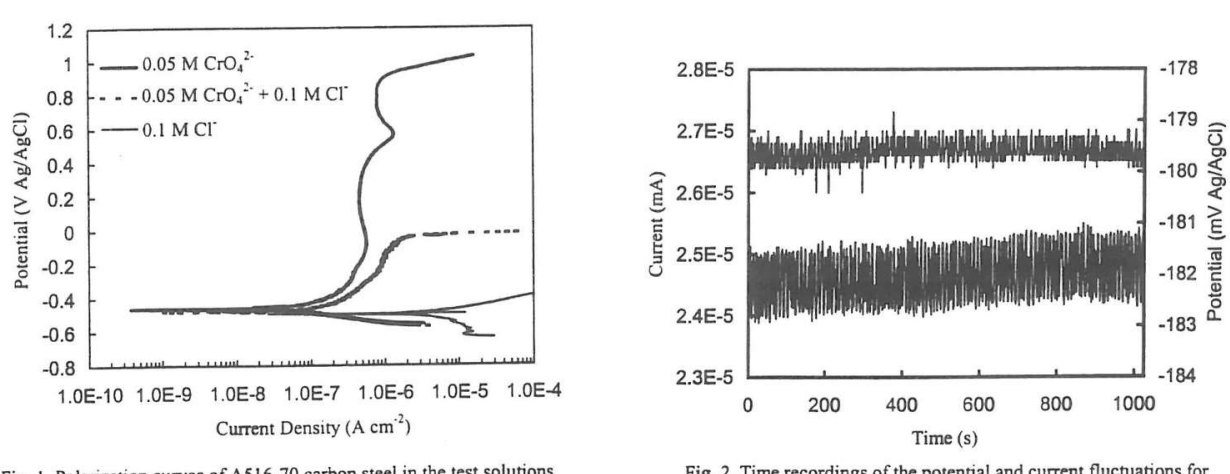

ig. 1. Polanzzation curves of ASt
Ast16-70 carbon steel in $0.05 \mathrm{M} \mathrm{Na}$ accos, solution. 
The electrochemical noise generated in this system mainly comes from the very slow dissolution of passivated carbon steel and the reduction of oxygen. The features of potential and current noise, which are shown in Fig. 2, consist of very frequent fluctuations with the amplitudes of potential and current of approximately $1.5 \mathrm{mV}$ and $1 \mathrm{nA}$, respectively. Before the experiments, the inherent noise of the measurement system was detected. The results indicated that the instrument noise also consists of frequent and stochastic potential and current fluctuations, with the amplitudes of potential and current fluctuations lower than $1 \mathrm{mV}$ and $0.5 \mathrm{nA}$, respectively.

The time records for potential and current noise were analyzed statistically by calculating noise resistance, $R_{n}$, and spectral noise resistance, $R_{s n}{ }^{0}$. The values of charge-transfer resistance, $R_{t}$, were determined by impedance measurements. The variations of $R_{n}, R_{s n}{ }^{0}$ and $R_{t}$ with the exposure time are plotted in Fig. 3. All three parameters were found to exhibit the same trend. After about six hours of immersion, the three variables fluctuated around $1.8 \times 10^{6} \Omega$, indicating that the stable passive state had been reached. For this corrosion system, $R_{n}, R_{s n}{ }^{0}$ and $R_{t}$ have very similar values although they are mathematically unrelated.

The spectral noise impedance, $R_{s n}(f)$, was determined by transforming the noise data from the time domain into the frequency domain by FFT. The comparison of $R_{\mathrm{sn}}(\mathrm{f})$ and the modulus of electrode impedance, $|\mathrm{Z}|$, determined by EIS measurements is shown in Fig. 4. Excellent agreement between both curves obtained by the two different techniques was observed at frequencies lower than $0.5 \mathrm{~Hz}$. Spectral analysis at a higher frequency range was not performed because of the limitation of sampling rate in electrochemical noise measurements as described above.

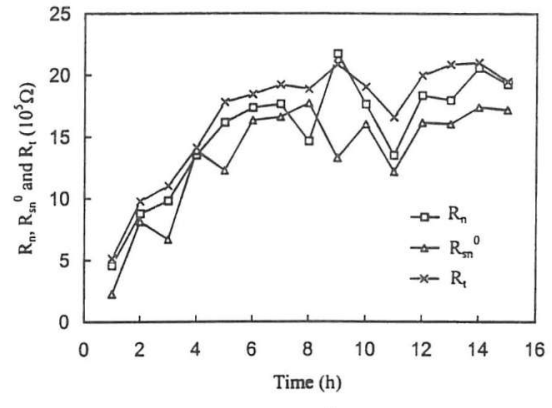

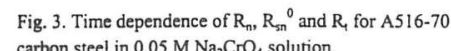

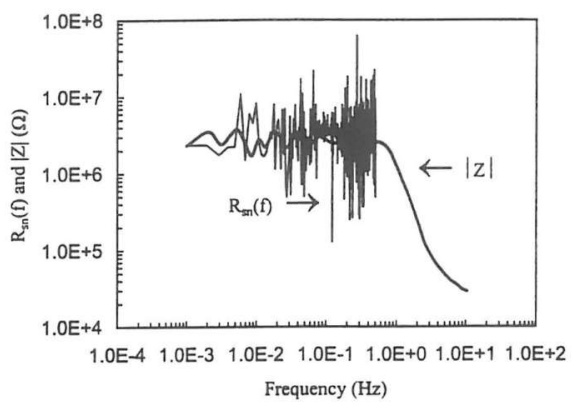

Fig. 4. $R_{m}(f)$ and $|z|$ for AS16-70 carbon steel

\section{Carbon steel in chloride solution}

From Fig. 1, it is clear that carbon steel electrode exposed to $0.05 \mathrm{M} \mathrm{NaCl}$ solution exhibit an active dissolution behavior consistent with a general corrosion system. Fig. 5 shows the potential and current noise recordings for this system. It can be seen that potential and current fluctuated with a lower frequency than was observed in the previous system discussed. However, the magnitude of background current (about $10^{-3} \mathrm{~mA}$ ) was much larger than that measured for the passive system $\left(10^{-5} \mathrm{~mA}\right)$, and the value of background potential, which was about $-646 \mathrm{mV}$, was more negative than the potential value of $-180 \mathrm{mV}$ under passive conditions. Consequently the carbon steel electrode dissolved with a very high rate in this system.

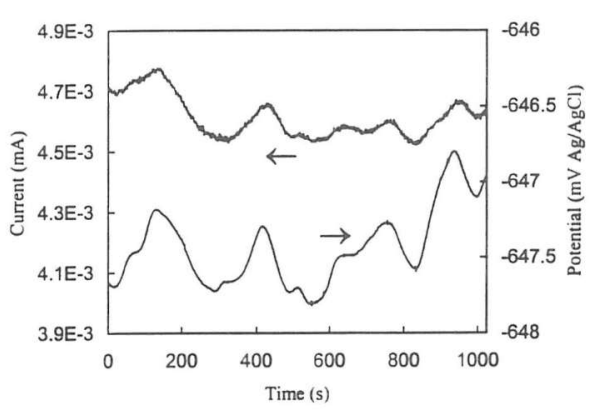

Fig. 5. Time recordings of the potential and current fluctuations
for A516-70 carbon steel in $0.05 \mathrm{M} \mathrm{NaCl}$ solution.

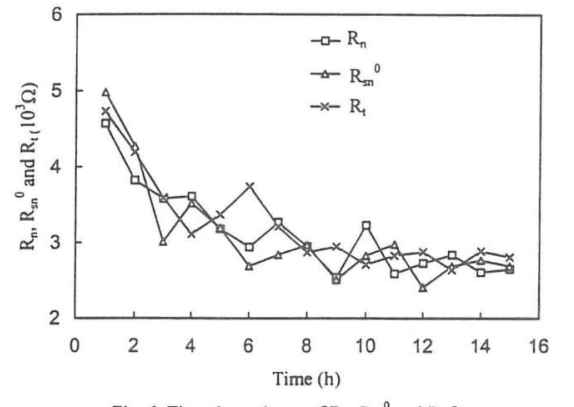

Fig. 6. Time dependence of $R_{n,} R_{s}{ }^{\circ}$ and $R_{\text {f for }}$
A516-70 carbon steel in $0.05 \mathrm{M}$ NaCl solution.
Comparison of $R_{n}, R_{s n}{ }^{0}$ and $R_{t}$ as a function of exposure time is shown in Fig. 6. It is seen that these parameters decreased with the time. After about six hours of immersion, all of the three variables tended 
towards a constant value and fluctuated around $2.6 \times 10^{3} \Omega$, three orders of magnitude less than tha under passive conditions. When the stable general corrosion state was attained, the $R_{n}, R_{s n}{ }^{0}$ and $R_{1}$ had very similar values.

The comparison of the spectral noise impedance, $R_{\mathrm{sn}}(\mathrm{f})$, and the modulus of electrode impedance, $|\mathrm{Z}|$, as a function of frequency is shown in Fig. 7. The two curves are very close to each other at frequencies lower than $0.5 \mathrm{~Hz}$.

Carbon steel in chromate solution containing chloride ions

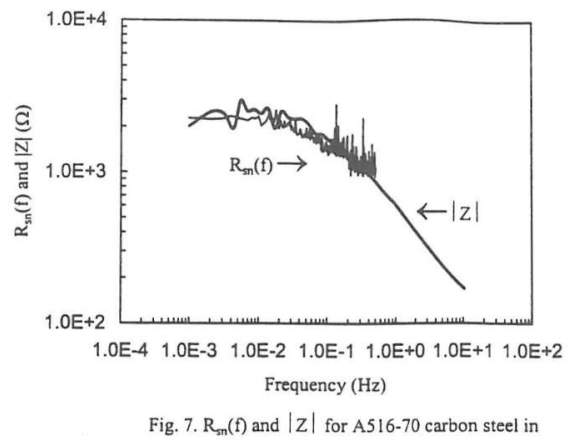

Fig. 7. $R_{m}(f)$ and $|z|$
$0.05 \mathrm{M} \mathrm{NaCl} \mathrm{solution.}$

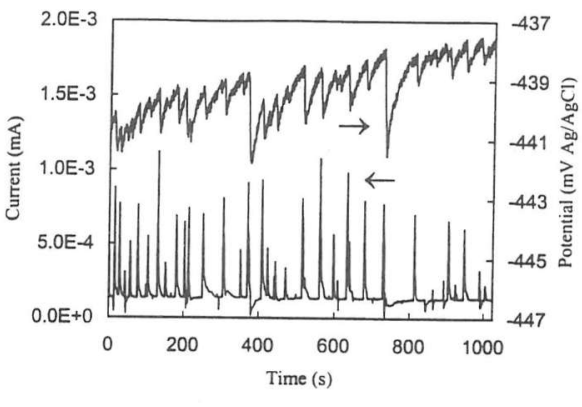

Fig. 8. Time record of the potential and current fluctuations for
AS16-70 carbon steel in $0.05 \mathrm{M} \mathrm{CrO}_{4}^{2}+0.1 \mathrm{MCl}$ solution.
It is well known that the addition of chloride ions to chromate inhibitor solution has a negative effect on the passivity of carbon steel [22]. As shown in Fig. 1, when the chromate solution contained $0.1 \mathrm{M} \mathrm{Cl}$, the passive potential range decreased to about $400 \mathrm{mV}$ and the passive current density also slightly increased. Fig. 8 shows the potential and current noise recordings after 10 hours of immersion. The typical transients, which were characterized by a rapid current rise and potential drop followed by slow recovery, appeared after a certain time of immersion. This type of transient is believed to indicate the initiation, growth and repassivation of metastable pits [28-30].

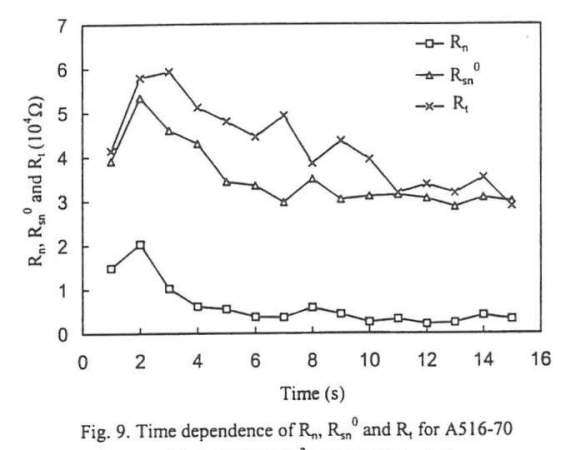

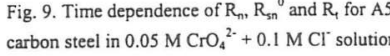

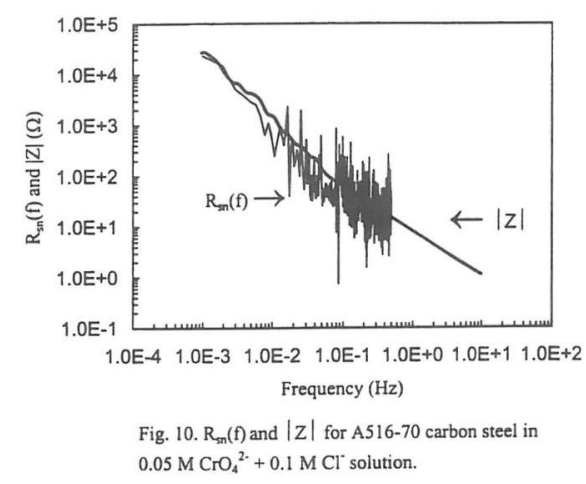

Fig. 9 shows the noise resistance, spectral noise resistance and charge-transfer resistance as a function of exposure time for $\mathrm{A} 516-70$ carbon steel in the $0.05 \mathrm{M} \mathrm{CrO}_{4}{ }^{2-}+0.1 \mathrm{M} \mathrm{Cl}$ solution. It was found that the time dependence of the three parameters was similar, that is, they all slightly decreased with the exposure time after two hours, indicating the degradation of passivity caused by metastable pitting. However, the numerical values of $R_{n}$ are much lower than $R_{s n}{ }^{0}$ and $R_{t}$, while the values of $R_{s n}{ }^{0}$ and $R_{t}$ are relatively close to each other.

Fig. 10 shows $R_{\text {sn }}(\mathrm{f})$ obtained from the potential and current PSD data, together with the modulus $|z|$ measured on the carbon steel specimens in $0.05 \mathrm{M} \mathrm{CrO}_{4}{ }^{2-}+0.1 \mathrm{M} \mathrm{Cl}$ solution. It was seen that $|\mathrm{Z}|$ coincided with the $R_{\text {sn }}(\mathrm{f})$ curve over the experimental frequency range. Different from the curves collected in a passive system (Fig. 4) and in a general corrosion system (Fig. 7), there was no low frequency plateau for both curves in Fig. 10.

\section{Discussion}

The experimental results of EN measured for three systems indicate that the spectral noise impedance $R_{\mathrm{sn}}(\mathrm{f})$ is equal to the modulus $|z|$ of the electrode impedance over the experimental frequency range. The results presented in this work were obtained from three systems with quite different noise sources: for carbon steel in chromate solution the principal source of EN in the passive system is the slow dissolution of passivated carbon steel; for carbon steel in chloride solution the EN mainly comes from the general corrosion of carbon steel; while for carbon steel in the $\mathrm{CrO}_{4}{ }^{2}-\mathrm{Cl}^{-}$solution, the noise source is pitting of carbon steel. Therefore, the agreement between $\mathrm{R}_{\mathrm{sn}}(\mathrm{f})$ and $|\mathrm{Z}|$ seems to be independent of the corrosion mechanism. The theoretical model developed by Bertocci et al. [14] predicted that the 
spectral noise impedance is proportional to the modulus of the electrode impedance, and with the proportional factor having the value of 1 if a "noiseless" reference electrode is used. The noise analysis performed on carbon steel in $\mathrm{CrO}_{4}{ }^{2-}$ and/or $\mathrm{Cl}^{-}$solutions confirm the theoretical predictions of thei model.

Once established that $R_{s n}(f)$ is equivalent to the $|z|$, it is possible to discuss the significance of noise resistance obtained by EN measurements. The relationship between $R_{n}$ and $R_{s n}(f)$ is [14]

$$
R_{n}=\left[\frac{\int_{f \min }^{f \max } I_{P S D}(f) R_{S n}(f)^{2} d f}{\int_{f \min } I_{P S D}(f) d f}\right]^{\frac{1}{2}}=\left[\frac{\int_{f \min }^{f \max } E_{P S D}(f) d f}{\int_{f \max } I_{P S D}(f) d f}\right]^{\frac{1}{2}}
$$

where $f_{\min }$ and $f_{\max }$ are defined by Eq. 1 .

For the $R_{\text {sn }}(f)$ (or $|z|$ ) vs. frequency curve with a long low frequency plateau, such as these shown in Fig. 4 and Fig. 7, the right side of Eq. 5 gives a value corresponding to the zero frequency limit of the spectral noise impedance (spectral noise resistance). This conclusion can be numerically verified. In a passive system, the values of $R_{n}$ and $R_{s n}{ }^{0}$ obtained from the time records in Fig. 3 after 6 hours of immersion (the system is in a steady state) range from $1.3 \times 10^{6}$ to $2.2 \times 10^{6} \Omega$, with a mean of $1.8 \times 10^{6} \Omega$, which approaches the $\mathrm{R}_{\mathrm{sn}}(\mathrm{f})$ and $|\mathrm{Z}|$ curves at frequency range of $10^{-3}$ to $0.5 \mathrm{~Hz}$, and approximates to the mean value of $2 \times 10^{6} \Omega$, in Fig. 4. In such a case, both $R_{n}$ and $R_{s n}{ }^{0}$ coincide with $R_{t}$, which is shown in Fig. 3. Therefore, the noise resistance and the spectral noise resistance provide an indication of the corrosion resistance of the material in a stable passive state. For the general corrosion system, the average noise resistance in the stable state is determined as about $2.8 \times 10^{3} \Omega$ from Fig. 6 . The mean spectral noise impedance at the frequency range of $10^{-3}$ to $2 \times 10^{-2} \mathrm{~Hz}$ is approximately $2.5 \times 10^{3} \Omega$, as shown in Fig. 7. Therefore, the noise resistance and the spectral noise resistance also provide an indication of the corrosion resistance of a general corrosion system.

However, there are some cases where $R_{n}$ might differ substantially from $R_{t}$ and $R_{s n}{ }^{0}$. This is wel illustrated by the measurements made in chromate/chloride solution as shown in Figs. 8, 9 and 10. Fig. 8 shows that the potential and current noise generated during metastable pitting in the time domain appears as short current bursts along with potential drops. Fig. 10 shows that the $\mathrm{R}_{\mathrm{sn}}(\mathrm{f})$ is frequency dependent over the experimental frequency range, and that there is no plateau in the low frequency range. According to Eq. 5, there is no reason for $R_{n}$ to be equal to the low frequency limit of the spectral noise impedance, $R_{s n}{ }^{0}$. In fact, $R_{n}$ is much lower than $R_{s n}{ }^{0}$ and $R_{t}$ as shown in Fig. 9. Therefore, the noise resistance can be used to only qualitatively, but not quantitatively, track the change of resistance of the electrode to pitting corrosion. The spectral noise resistance is consistent with the charge-transfer resistance and can be used to indicate the corrosivity under three conditions: passivity, general corrosion and pitting.

\section{Conclusions}

The results of the EN measurements and EIS analysis carried out on A516-70 carbon steel in chromate and/or chloride solutions show that the spectral noise impedance is equivalent to the modulus of the electrode impedance. The relationship between them is independent of the corrosion conditions (passivity, general corrosion and pitting). The noise resistance coincides with the spectral noise resistance and the charge-transfer resistance only under conditions of passivity or general corrosion, where the noise resistance provides an indication of the corrosion resistance of the material under study During pitting corrosion, the noise resistance is much lower than the spectral noise resistance and charge-transfer resistance, and can be used to track only qualitatively, but not quantitatively, the change of resistance of the system to pitting corrosion. The spectral noise resistance is consistent with the charge-transfer resistance and can be used to indicate the corrosivity under three conditions: passivity, general corrosion and pitting.

\section{Acknowledgments}

This research is supported by Environmental Science and Technology Alliance Canada. Helpfu discussions with W. Karch in Dow Chemical Canada Inc. are highly appreciated.

\section{References}

[1] J.L. Dawson, Electrochemical Noise Measurement for Corrosion Applications, J.R. Kearns, J.R Scully, P.R. Roberge, D.L. Reichert and J.L. Dawson, Editors, p. 3, ASTM STP 1277, West Conshohocken, PA (1996). 
[2]. C. Gabrielli, F. Huet and M. Keddam, Electrochemical and Optical Techniques for the Study and Monitoring of Metallic Corrosion, M.G.S. Ferreira and C.A. Melendres, Editors, p. 135, Kluwer Academic Publishers, Netherlands (1991).

[3] U. Bertocci and F. Huet, Corrosion 51 (1995) 131.

[4] C. Liu, D.D. Macdonald, E. Medina, J.J. Villa and J.M. Bueno, Corrosion 50 (1994) 687.

[5] A. Legat and V. Dolecek, Corrosion 51 (1995) 295.

[6] N.J. Laycock, T. Stewart and R.C. Newman, Corros. Sci. 39 (1997) 1620.

[7] T.T. Lunt, B. Wu, J.R. Scully and J.L. Hudson, J. Electrochem. Soc. 144 (1997) 1620.

[8] F. Mansfeld and H. Shih, J. Electrochem. Soc. 135 (1988) 1171.

[9] F. Mansfeld and H. Xiao, J. Electrochem. Soc. 140 (1993) 2205.

[10]H. Xiao and F. Mansfeld, J. Electrochem. Soc. 141 (1994) 2332.

[11]D.L. Reichert, Electrochemical Noise Measurement for Corrosion Applications, J.R. Kearns, J.R. Scully, P.R. Roberge, D.L. Reichert and J.L. Dawson, Editors, p. 79, ASTM STP 1277, West Conshohocken, PA (1996).

[12] A. Legat and C. Zevnik, Corros. Sci. 35 (1993) 1661.

[13] C.T. Chen and B.S. Skerry, Corrosion 47 (1991) 598.

[14]U. Bertocci, C. Gabrielli, F. Huet and M. Keddam, J. Electrochem. Soc. 144 (1997) 31.

[15]U. Bertocci, C. Gabrielli, F. Huet, M. Keddam and P. Rousseau, J. Electrochem. Soc. 144 (1997)

[16]R.A. Cottis, S. Turgoose and J. Mendoza-Flores, Electrochemical Noise Measurement for Corrosion Applications, J.R. Kearns, J.R. Scully, P.R. Roberge, D.L. Reichert and J.L. Dawson, Editors, p. 93, ASTM STP 1277, West Conshohocken, PA (1996).

[17]R. A. Cottis and S. Turgoose, Materials Science Forum 192-4 (1995) 663

[18] J.W. Cooly and J.W. Tukey, Mathematics of Computation 19 (1965) 297.

[19] W.H. Press, B.P. Flannery, S.A. Teukolsky and W.T. Vettering, Numerical Recipes in Pascal, p. 217, Cambridge University Press, Cambridge, UK (1989).

[20]R.G. Kelly, M.E. Inman and J.L. Hudson, Electrochemical Noise Measurement for Corrosion Applications, J.R. Kearns, J.R. Scully, P.R. Roberge, D.L. Reichert and J.L. Dawson, Editors, p. 101, ASTM STP 1277, West Conshohocken, PA (1996).

[21]J.L. Dawson, D.M. Farrel, P.J. Aylott and K. Hladky, Corrosion/89, Paper no. 31, NACE, Houston, TX (1989).

[22] G.P. Bierwagen, J. Electrochem. Soc. 141 (1994) L155.
[23]F. Mansfeld and H. Xiao, J. Electrochem. Soc. 141 (1994) 1403.

[24]F. Huet, J. Electrochem. Soc. 142 (1995) 2861.

[25] J.F.Chen and W.F.Bogaerts, Corros. Sci. 37 (1995) 1839.

[26]E. McCafferty, J.Electrochem.Soc. 137 (1990) 3731.

[27] C. Monticelli, G. Brunoro, A. Frigani and G. Trabanelli, J. Electrochem. Soc. 139 (1992) 693

[28]S.T. Pride, J.R. Scully and J.L. Hudson, Electrochemical Noise Measurement for Corrosion

Applications, J.R. Kearns, J.R. Scully, P.R. Roberge, D.L. Reichert and J.L. Dawson, Editors, p.

307, ASTM STP 1277, West Conshohocken, PA (1996).

[29] G. S. Frankel, L. Stockert, F. Hunkeler and H. Bohni, Corrosion 43 (1987) 429.

[30] S. T. Pride, J. R. Scully and J. L.Hudson, J. Electrochem. Soc. 141 (1994) 3028.
Submitted $10^{\text {th }}$ September 2000

Accepted 30th November 2000 